\title{
Etnográfica
}

Revista do Centro em Rede de Investigação em

Antropologia

vol. $22(2) \mid 2018$

Vol. $22(2)$

\section{Etnografía móvil, entre el sombreado y el acompañamiento: notas a partir del estudio de la movilidad cotidiana en la Región Metropolitana de Barcelona (RMB)}

Mobile ethnography, between accompaniment and shadowing: notes from the study of daily mobility in the Metropolitan Area of Barcelona

\section{Joan Josep Pujadas}

\section{OpenEdition}

\section{Journals}

\section{Edición electrónica}

URL: https://journals.openedition.org/etnografica/5531

DOI: 10.4000/etnografica.5531

ISSN: 2182-2891

Editor

Centro em Rede de Investigação em Antropologia

Edición impresa

Fecha de publicación: 1 junio 2018

Paginación: 361-386

ISSN: 0873-6561

Referencia electrónica

Joan Josep Pujadas, «Etnografía móvil, entre el sombreado y el acompañamiento: notas a partir del estudio de la movilidad cotidiana en la Región Metropolitana de Barcelona (RMB)», Etnográfica [En línea], vol. 22 (2) | 2018, Publicado el 07 julio 2018, consultado el 19 enero 2022. URL: http:// journals.openedition.org/etnografica/5531; DOI: https://doi.org/10.4000/etnografica.5531

\section{(c) (†) 8}

Etnográfica is licensed under a Creative Commons Attribution-NonCommercial 4.0 International License. 


\section{Etnografía móvil, entre el sombreado y el acompañamiento: notas a partir del estudio de la movilidad cotidiana en la Región Metropolitana de Barcelona (RMB)}

\section{Joan Josep Pujadas}

El estudio de la movilidad cotidiana en la RMB ha significado el replanteamiento de los instrumentos metodológicos necesarios para estudiar este fenómeno. La contextualización localizada de los sujetos bajo análisis ha sido substituida por el análisis de sus movilidades, de su direccionalidad y del significado que poseen en su vida cotidiana. Esta etnografía móvil se ha apoyado esencialmente en el instrumento del acompañamiento de los viajes y los desplazamientos de las personas para ir al trabajo, a estudiar o a solucionar todo el universo de necesidades surgidas de la vida personal, doméstica y reproductiva. Este énfasis en el acompañamiento desplaza la perspectiva analítica del shadowing, al considerar esta técnica demasiado intrusiva.

PALABRAS-CLAVE: movilidad, metodología, etnografía móvil, acompañamiento, sombreado.

Mobile ethnography, between accompaniment and shadowing: notes from the study of daily mobility in the Metropolitan Area of Barcelona - The study of the daily mobility in the Metropolitan Area of Barcelona implied rethinking the methodological instruments necessary to study this phenomenon. The localized contextualization of the subjects under analysis has been replaced by the analysis of people's mobilities, their directionality and the meaning they assume in the subjects' daily lives. This mobile ethnography has been essentially based on an instrument consisting of the accompaniment of travels and movements of people going to work, to school or solving the whole universe of needs arising from personal, domestic and reproductive life. This emphasis on accompaniment shifts the analytical perspective of the shadowing, considering this technique too intrusive.

KEYWORDS: mobility, methodology, mobile ethnography, accompaniment, shadowing.

PUJADAS, Joan Josep (joanjosep.pujadas@urv.cat) - Universitat Rovira i Virgili, Tarragona, España. 
LA MOVILIDAD HUMANA, ADEMÁS DE CONSOLIDARSE COMO EL OBJETO de estudio de un número creciente de investigaciones, constituye una dimensión poliédrica de la realidad social y del estilo de vida de las poblaciones metropolitanas que ha ido redefiniendo las formas de vinculación de las personas con el territorio. ${ }^{1}$ El radio de acción de las actividades cotidianas de las personas se va ensanchando y sus "espacios de vida" son cada vez más extensos y policéntricos (Courgeau 1988; Alberich González 2010). El sentido de lugar y las identificaciones territoriales han sido sometidos a unas presiones que han tendido a transformarlas. Los escenarios que enmarcan las secuencias de la actividad cotidiana de las personas no son solamente múltiples, si no cambiantes. Para muchos no queda casi nada de la vieja vinculación de las personas con sus lugares, entendidos como espacios acotados de relaciones sociales múltiples.

Consecuentemente a estas transformaciones en la organización social y territorial, las aproximaciones etnográficas a estas nuevas realidades han experimentado notables modificaciones en las últimas décadas, desde las propuestas sobre la etnografía multisituada (Marcus 1995), hasta las críticas al nacionalismo metodológico asociadas a la necesidad de adoptar una mirada trasnacional (Beck 2004; Levitt e Glick Schiller 2004), las propuestas sobre las formas desiguales de acceso a la movilidad (Chicoine 1998; Imilán, Jirón y Iturra 2015; Jirón 2007) o la existencia de regímenes de movilidad (Salazar 2010; Glick Schiller y Salazar 2013), que comporta el reconocimiento de que los procesos de globalización despliegan, al mismo tiempo, estrategias de eliminación de fronteras y de imposición de otras nuevas.

Estudiar la movilidad cotidiana en la Región Metropolitana de Barcelona (RMB) requirió de la búsqueda de una nueva mirada y de unas técnicas etnográficas específicas para captar aquellos fenómenos que definen la vida social metropolitana: movilidad, simultaneidad, fragilidad y multi-territorialidad. El sombreado (shadowing) constituye, junto a la observación directa, la técnica central de nuestra etnografía en/del espacio de vida de los urbanitas (Czarniawska 2007; Jirón 201 1; Novoa 2015).

\section{LAS UNIDADES DE ANÁLISIS EN LAS ETNOGRAFÍAS URBANAS}

La etnografía urbana ha poseído a lo largo de su trayectoria multisecular tres anclajes fundamentales, que le han servido de rasgos definidores: (1) situarse en escenarios urbanos territorialmente delimitados; (2) abordar relaciones

l Este trabajo forma parte del proyecto de investigación titulado "Movilidad y vida cotidiana en Cataluña: etno-geografía de los espacios y tiempos metropolitanos", financiado por la Dirección General de Investigación y Gestión del Plan Nacional de I+D+i del Ministerio de Economía y Competitividad, Gobierno de España (ref. CSO2012-35425). 
sociales de tipo comunitario; y (3) operar "desde dentro" de la unidad de análisis a partir de la empatía entre observador y observados, usando técnicas participativas.

Existen, sin embargo, en los estudios urbanos, otras tradiciones, no hegemónicas, que ponen en cuestión estos tres supuestos. Por un lado, la perspectiva del observador curioso y distanciado del "flâneur", propuesta por Benjamin (2005) y, por el otro, la "deriva" o el paseo ininterrumpido de un observador que se deja llevar "por las solicitaciones del terreno y por los encuentros que a él corresponden" (Debord 1999 [1958]). ${ }^{2}$

De la narrativa centrada en objetos particulares, situados y centrados, se pasa a visiones caleidoscópicas, complejas y deslocalizadas de sujetos que transitan por mundos superpuestos que convergen en las ciudades. Lugar, comunidad y enraizamiento dan paso a aproximaciones especulares sobre lugares, tránsitos, y encuentros. Desde el dominio de la estructura y de la solidez aparente de instituciones como familia, barrio o comunidad se transita hacia los dominios de la movilidad, la fluidez, la deslocalización y las identidades múltiples e híbridas (García-Canclini 1989; Hannerz 1996).

El fenómeno más relevante que debemos tomar en cuenta es el cambio de escala que ha experimentado la vida de las personas en el proceso que arranca en las últimas etapas de la ciudad industrial y que nos lleva hasta la situación actual, caracterizada por la deslocalización residencial y empresarial hacia áreas suburbanas, conectadas pero cada vez más alejadas de los centros urbanos (Mieszkowski y Mills 1993; Brueckner 2000). Hasta hace algo menos de un siglo los desplazamientos de casa al trabajo de los obreros industriales se producían a pie, en bicicleta o en tranvía. Sin embargo, hoy en día transitar por las grandes conurbaciones metropolitanas requiere de complejos sistemas de transporte multi-modal, así como de unos costes de locomoción, medidos en dinero y en tiempo de desplazamiento, que se convierten en obstáculos a la movilidad para extensas capas de la población (Sheller y Urry 2006; Franquesa 2011).

La escala en que se desarrollaron la mayor parte de los estudios "clásicos" de la etnografía urbana (Ware 1935; Whyte 1943; Bott 1957; Mayer 1961; Abu-Lughod 1961; Hannerz 1969; Butterworth 1962) fue el barrio o el enclave étnico urbano como unidad de análisis. Richard Fox (1977), evaluando las aportaciones de la subdisciplina, a lo largo de los años 1960 y 1970 consideraba que los etnógrafos de la ciudad se habían concentrado en dos objetos:

2 En los últimos años se registra un interés significativo por revisitar la obra de Walter Benjamin y, especialmente, por la figura del flanêur que, a pesar de su extracción literaria y de ficción, aporta una perspectiva de observador atento de la ciudad, en su deambular sin rumbo. Constituye, pues, una inspiración para las nuevas exigencias de la etnografía de la movilidad (Jenks y Neves 2000; Rocha y Eckert 2003; Prysthon 2007; Durán Segura 201 1; Santos, Ferreira y Silva 2014). Algo parecido puede decirse de la práctica de la "deriva”, propuesta por Guy Debord y la Internacional Situacionista (Durán Segura 2011 ; Pellicer, Rojas y Vivas-Elias 2012; Pellicer, Vivas-Elias y Rojas 2013). 
los "procesos de urbanización” (Bascom 1955; Mitchell 1956; Mayer 1961; Epstein 1967; Little 1974) y los "estudios sobre pobreza urbana" (Lewis 1961, 1966; Valentine 1968; Marks 1991), dejando de lado un enfoque más general y holístico, como el "urbanismo", entendido a la manera de Wirth (1938) o Park (1952), como conjunto de formas de vida urbana.

La mirada hacia el urbanismo y la ruptura con los barrios, entendidos como lugares comunitarios auto-contenidos, venía ya marcada por algunos de los estudios precursores realizados por los etnógrafos de Manchester en Zambia, especialmente en la región del Copperbelt. Su estrategia era la realización de estudios de caso transversales que permitían, a partir del análisis intensivo de una situación particular, presentar cuadros más amplios de interpretación sobre la estructura social y los conflictos que se desarrollaban en las ciudades africanas de mediados del siglo XX (Gluckman 1958). Los trabajos de Mitchell (1956) sobre el "ritual étnico" de la danza kalela en la ciudad minera de Luanshya, y el de Epstein (1958) sobre la destribalización de las categorías de prestigio social y la aparición de una conciencia obrera entre los mineros de la misma ciudad, constituyen una de las aportaciones epistemológicas más novedosas para articular lo micro y lo macro, desde fenómenos particulares a marcos sociales amplios, que dan sentido y permiten la interpretación de las particularidades observadas y descritas etnográficamente.

Directamente vinculados a la Escuela de Manchester aparecen algunos trabajos pioneros en el análisis de redes sociales (Barnes 1954; Mitchell 1969; Bott 1957; Boissevain y Mitchell 1973). La utilidad del análisis de redes para la investigación urbana es indiscutible, ya que permite trascender las esferas de la familia y del parentesco, a la vez que las estrechas y cada vez más difusas relaciones localizadas de tipo barrial o comunitario. Permite, por otro lado, situar las interacciones personales en el marco de las instituciones, divisorias y segmentos en que se organiza cada sociedad.

Como recurso etnográfico, el análisis de las redes personales nos proporciona la posibilidad de establecer y, hasta cierto punto, medir las conexiones y anclajes que cada sujeto establece en los diferentes ámbitos de actividad y sociabilidad que desarrolla. Como señala J.L. Molina González (2005: 71), en las redes personales podemos observar interacciones institucionalizadas (familiares, de amistad, laborales, políticas o étnicas) en el marco de estructuras sociales estratificadas que las influyen, condicionan o las hacen posibles. El análisis de redes, como señala el mismo autor, nos permite avanzar en la comprensión de los fenómenos sociales de rango intermedio o meso; esto es, aquel ámbito en el que se producen simultáneamente interacciones de tipo personal e institucional.

Tras muchas décadas en que el canon etnográfico consistía en estudios intensivos de larga duración en unidades de observación de pequeña escala, realizados en lugares remotos, en el último cuarto del siglo XX empezaron a 
predominar otros enfoques, otras aproximaciones, en que las prácticas etnográficas se hicieron más ambiciosas, a la vez que heterodoxas. Lomnitz (1977), por ejemplo, para responder a la pregunta de cómo pueden sobrevivir en condiciones de extrema precariedad los vecinos de Cerrada del Cóndor en Ciudad de México, acude no solamente a explicaciones endógenas sobre los mecanismos de solidaridad y entre-ayuda dentro la comunidad vecinal, sino a las actividades que los vecinos desarrollan fuera de la comunidad y a las redes de compadrazgo que se van tejiendo como estrategia para salir de la precariedad. Sin la articulación de la barriada con la ciudad, muchos de los fenómenos observados a nivel micro carecerían de un marco interpretativo.

La monografía de Safa Barraza (1998) sobre Coyoacán (Ciudad de México) constituye una aportación que, sin abandonar totalmente un anclaje etnográfico localizado, se sitúa en la encrucijada del policentrismo y las mediaciones. Su unidad de análisis son los imaginarios y las prácticas de lugar en el distrito urbano de Coyoacán. ${ }^{3}$ Se trata de una realidad compleja que agrupa barrios obreros y de clase media, áreas centrales y monumentales en que habitan algunos de los sectores de la alta burguesía capitalina y pueblos habitados por indígenas nahuas, junto a amplias capas de población mestiza, subalterna y marginada, que ocupan especialmente algunas de las colonias de la unidad administrativa. Sus aportaciones se centran en el análisis de los imaginarios de sus habitantes y visitantes, así como en las formas de articulación de las prácticas cotidianas, algunas de las cuales entran en colisión. Se trata, de hecho, de un análisis multi-situado, que la lleva de un enclave a otro, de un espacio público a otro. El distrito urbano y la ciudad entera constituyen el marco general en el que se encuadran e interpretan los hallazgos etnográficos: conflictos, movilizaciones vecinales, dinámicas residenciales, enclaves turísticos y formas diferenciales de vivir y significar los lugares.

Estos dos trabajos se sitúan, claramente, en un nivel meso de análisis. Mientras Lomnitz explora las alianzas y redes sociales de los habitantes de Cerrada del Cóndor, Safa Barraza explora las conexiones entre los diferentes enclaves de su unidad de análisis, presentando las diferentes formas de mediación en los conflictos entre residentes con las autoridades de la Delegación y con otros actores sociales externos. Las unidades de observación dejan de ser vistas y narradas como universos autocontenidos. La perspectiva relacional que defendía Hannerz (1986 [1980]) está ya presente en estos trabajos, marcando una pauta bien definida hacia el futuro.

En la década de 1990 se producen cambios de rumbo cruciales a nivel teórico y epistemológico, tanto en el ámbito de la economía, la historia o en los estudios culturales, así como en la misma antropología, que nos permiten

3 Con más de $54 \mathrm{~km}^{2}$ de superficie y 630.000 habitantes, la Delegación Coyoacán está constituida por 8 barrios, 5 pueblos y 30 colonias. Más que un distrito urbano, Coyoacán es una ciudad. 
entender la orientación que van a adoptar los nuevos enfoques metodológicos, que transitan desde las etnografías localizadas hasta los análisis multisituados. Se produce, pues, un relevante cambio de escala en la definición de las unidades de análisis. Sin abandonar del todo la escala local, la etnografía busca espacios para operar en los niveles meso y macro. El propio Marcus (1995) resume la herencia intelectual que conduce a su exitosa formulación sobre la "etnografía multisituada", que consiste, antes que nada, en mapear un territorio no pretendiendo una representación holística de su totalidad, sino la identificación de las fronteras de un fenómeno, para poder establecer conexiones e identificar procesos entre las diferentes localizaciones que entran dentro del campo de análisis (Marcus 1995: 96-99).

El trabajo de A. Appadurai (1990, 1991 [1986]) sobre la economía cultural global aporta una perspectiva multilocal que se inserta en dinámicas transnacionales y que marca la evidencia del sinsentido que tiene situar fenómenos culturales en una esfera local, cuando la circulación y su proceso de transmisión a una escala global son las claves para entender los fenómenos en estudio. El trabajo de Appadurai bebe de fuentes como el trabajo de S. Mintz (1985) sobre la historia cultural del azúcar, que muestra la técnica de "seguir los objetos" desde una perspectiva de economía política, o como el influyente trabajo del historiador I. Wallerstein (1974) sobre el sistema mundo. Marcus también sitúa algunas de las más notables contribuciones teóricas contemporáneas en el marco de las críticas al sistema capitalista y a los procesos de disolución y fragmentación del orden mundial moderno. Se trata de todo un conjunto de aportaciones que se sitúan en el ámbito global, aportando perspectivas y conceptos para la comprensión del nuevo desorden posmoderno: posfordismo, compresión del espacio-tiempo, especialización flexible (Harvey 1998 [1989]), fin del capitalismo organizado (Lash y Urry 1998), globalización (Featherstone 1990; Hannerz 1992), junto a la perspectiva del transnacionalismo (Glick Schiller, Basch y Szanton Blanc 1992).

En un mundo globalizado, caracterizado por el comercio a larga distancia, por la circulación constante de capitales y por la movilidad creciente de las personas - a corta, media y larga distancia -, se ha operado una compresión de la relación entre espacio y tiempo (Harvey 1998 [1989]). La fluidez, más que la estructura, caracteriza nuestras vidas y trayectorias, también nuestra relación con los objetos, las personas y las relaciones institucionalizadas: familia, trabajo o participación social. Estas perspectivas se conectan y dialogan con otras aportaciones, pertinentes para nuestra fundamentación, como las de "sociedad líquida" (Bauman 2003 [2000]) o "sociedad del riesgo" (Beck 1998 [1986]). Ambas perspectivas nos presentan unos marcos sociales llenos de incertezas en el que las trayectorias personales y los proyectos de vida están marcados por la provisionalidad, la fragilidad y los cambios de rumbo. Éste mundo cambiante y en proceso permanente de reestructuración contrasta vivamente con la solidez 
monótona del mundo de vida de los jóvenes escolares de Birmingham estudiados por Willis en su ensayo sobre la cultura contraescolar - unos jóvenes que desatienden su formación académica, que consideran superflua, pues tienen la certeza de que les aguarda un futuro laboral en las mismas fábricas en las que trabajan o trabajaron sus padres y abuelos (Willis 1988 [1978]).

Desde la época de Malinowski, la comunidad local fue no solamente unidad de observación, sino unidad de análisis. Un universo autocontenido. Las comunidades humanas en las narrativas etnográficas clásicas, cual enjambres de abejas, nos mostraban sujetos clónicos, poseedores de unos valores, conocedores de unas reglas sociales y, en definitiva, seres humanos sometidos en sus comportamientos al imperio de esa entidad amorfa y omnipresente denominada cultura. En las etnografías clásicas, las personas, reducidas en su función comunicativa con el etnógrafo a meros "informantes", eran intercambiables unas con otras. La etnografía, en este sentido, ha despojado de individualidad a la persona, ha desdibujado su perfil autónomo, único y subjetivo para convertirlo en parte de un cuadro social de posiciones fijas, pensado en términos de solidez, homogeneidad y coherencia. La etnografía de hoy en día, sin embargo, no puede operar ni con el concepto de comunidad en singular - pues son varias las comunidades o nódulos de relaciones en que se inserta la cotidianidad de cada sujeto - ni con la categoría de cultura, entendida al estilo clásico como un atributo compartido de manera privativa y específica por un grupo social y que, como fenómeno social, posee fronteras bien definidas, que permiten caracterizar a cada persona como perteneciente a un grupo y solamente a uno (Pujadas 2011).

\section{LA MOVILIDAD DENTRO DE LA RMB}

Como se ha señalado al inicio del texto, trabajamos con la categoría "espacio de vida", como forma de aprehender de manera estratificada la extensión territorial en la que se desenvuelven las actividades cotidianas de las personas que habitan en la Región Metropolitana de Barcelona y en el Camp de Tarragona. ${ }^{4}$ Como señalan Alberich González y Roquer Soler (2014: 490), aproximadamente el 50\% de la población empleada en Cataluña trabaja en el mismo municipio de residencia. Por otro lado, los niveles de autocontención laboral se disparan en las ciudades capitales (Barcelona, 84\%; Tarragona, 79\%; Girona, 60\%), mientras que la movilidad crece en los territorios colindantes a las principales ciudades. La "movilidad cotidiana", entendida como los

4 Nuestra unidad territorial de análisis se centra en todo el sector central de la franja litoral catalana, con excepción de las regiones septentrional (Girona) y meridional (Terres de l'Ebre). Hemos elegido, por tanto, el territorio más poblado y el que presenta la mayor parte de la actividad industrial, logística y de servicios de Cataluña. 
desplazamientos pendulares diarios que se realizan entre el lugar de residencia y el lugar de trabajo (o estudio, ocio o consumo) y que presuponen desplazamientos intermunicipales, ni afecta a toda la población, ni lo hace por igual. ${ }^{5}$

Reconocemos que, si bien el policentrismo y la multilocalidad forman parte de la cotidianidad de un número creciente de ciudadanos, el sedentarismo y la inmovilidad siguen constituyendo todavía la experiencia diaria de la mayoría de personas, al menos en la RMB. Existen perfiles definidos que marcan la mayor o menor tendencia a la movilidad cotidiana. Alberich y Roquer, que elaboraron los resultados de la "Enquesta de Condicions de Vida i Hàbits de la Población 2011”, concluyen lo siguiente:

"Los resultados obtenidos muestran que las variables más determinantes son, por este orden, el sexo (los hombres tienen mayor probabilidad de realizar un desplazamiento intermunicipal por motivos de trabajo), la edad (cuanto menor es la edad, mayor es la movilidad) y el nivel de estudios (mayor movilidad entre los niveles formativos más bajos), mientras que el estado civil no se muestra como una variable significativa" (Alberich González y Roquer Soler 2014: 496). ${ }^{6}$

Este dibujo estadístico general de las principales variables que influyen en la movilidad dentro del área geográfica explorada ha contribuido a la selección de casos en nuestro trabajo etnográfico. Por lo que respecta a la variable edad, en nuestra muestra hemos excluido a las personas menores de 18 años y a las mayores de 65. La variable género hemos procurado mantenerla equilibrada, mientras que, por razones logísticas, en la variable nivel de estudios hemos primado el estudio del personal universitario (profesorado, alumnado y personal administrativo y de servicios), aprovechando la facilidad de acceso al personal de los dos campus en que se ubicaban los etnógrafos, miembros del equipo de investigación: Tarragona (URV) y Bellaterra (UAB). No hemos elidido, sin embargo, el estudio de casos que ilustran un amplio abanico de profesiones y niveles socio-económicos. En total, hemos reunido una muestra de 72 personas: dentro del ámbito universitario hemos estudiado al profesorado (12), personal administrativo (7), estudiantes (22), personal subalterno (2); junto a ellos, profesionales con estudios superiores (9), servicio doméstico y atención a las personas (3), empleados de banca (2), administrativos (3), otros profesores (4), mecánicos (2) y bombero (1); a estas personas hay que añadir a los

5 Junto a la creciente movilidad de la población, existe también un fenómeno de inmovilidad que constituye un indicador de segregación social y económica.

6 Los demógrafos Joan Alberich González y Santiago Roquer Soler forman parte del equipo de investigación del mencionado proyecto "Movilidad y vida cotidiana en Cataluña" (ref. CSO201235425), financiado por Dirección General de Investigación y Gestión del Plan Nacional de I+D+i del Ministerio de Economía y Competitividad. 
miembros de un grupo de sociabilidad ferroviaria (5) al que hemos seguido de forma periódica en sus desplazamientos a lo largo de siete meses. ${ }^{7}$

Esta muestra ha primado la observación de la movilidad hacia o desde Tarragona. En total, 42 personas viajan desde Tarragona, Barcelona, o alguna de las estaciones aledañas o intermedias a éstas en ambas direcciones. Nueve personas más viajan diariamente en coche desde diferentes poblaciones del Camp de Tarragona o Terres de l'Ebre hacia Tarragona. En segundo lugar, hemos estudiado los accesos a Bellaterra, tanto desde Barcelona (4), desde otras poblaciones litorales cercanas a Barcelona (5) o desde otras poblaciones del Vallès Occidental, cercanas a Bellaterra (5). Otras 7 personas, finalmente, se desplazan diariamente en la línea de alta velocidad desde o hacia Lleida, Tarragona, Barcelona o Girona.

En relación a los medios de transporte utilizados en los desplazamientos, de las 72 personas estudiadas, la mayoría son usuarios de transporte público, especialmente aquellos que van desde y hacia poblaciones con servicios ferroviarios de alta frecuencia de paso. Sin embargo, hemos complementado el análisis con 19 casos en que se usa el vehículo privado, que se corresponden con los clusters de Tarragona (URV) y Bellaterra (UAB) con sus respectivas poblaciones aledañas. ${ }^{8}$ Hemos analizado también los abundantes casos de itinerarios multimodales, que suponen el uso de vehículo privado o transporte público de corta distancia antes de usar el ferrocarril para un desplazamiento de media distancia.

Nuestra etnografía de la movilidad en la RMB ha operado a diferentes niveles. En primer lugar, la observación de campo en las fases iniciales de la investigación utilizó la técnica de la deriva, acompañada de fotografía y video, en los lugares de tránsito: estaciones ferroviarias, estaciones de autobuses, salas de espera, andenes, tiendas y cafeterías de estaciones de tren y bus y en estaciones de servicio de autopistas (Pink 2008; Spinney 2011). Allí descubrimos la importancia que tienen estos lugares para la realización de comidas rápidas y para el aprovisionamiento. Por otro lado, existen algunas estaciones de servicio en las autopistas catalanas que contienen verdaderos supermercados donde los transeúntes pueden realizar su aprovisionamiento periódico de alimentos, más allá de compras puntuales. Entre las cosas que fijaron nuestra atención en este deambular iniciático quiero destacar fenómenos como la burbuja de vacío alrededor de los usuarios de dispositivos móviles, concentrados en su pantalla y aislados del entorno, en las salas de espera. Otra observación, que a lo largo del tiempo hemos ido confirmando, es que la lectura de libros en las salas de

7 Las actividades profesionales de estas cinco personas son: militar (1), administrativo (2), cuidados personales (1), funcionario (1). El grupo está compuesto por cuatro mujeres y un hombre.

8 No debemos olvidar que, en buena medida, la "era de la movilidad" (mobility turn) es, antes que nada, una era de auto-movilidad (Urry 2004, 2008; Merriman 2009). 
espera y en los medios de transporte es un atributo casi exclusivamente femenino. Entre los grupos de personas que veíamos charlando, de acuerdo a su aspecto, vestido y edad, nos planteaban si se trataba de compañeros de trabajo o de estudio que viajaban juntos o bien si se trataba de relaciones generadas en los viajes. Esta pista la fuimos siguiendo hasta localizar a un grupo de amistad ferroviaria del que hablaremos más adelante.

Hemos utilizado técnicas clásicas de investigación, como la entrevista en profundidad, si bien en nuestro caso, más allá del énfasis en la narración de la movilidad, hemos enfocado estas entrevistas con profundidad temporal y biográfica. A este formato de entrevistas lo hemos denominado entrevistas en profundidad de orientación biográfica (ЕРOB)..$^{9}$ Una parte de las entrevistas individuales (38) han sido filmadas íntegramente y, junto al repertorio de fotografías y filmaciones adicionales de viajes, desplazamientos e itinerarios, conforman un material que nos ha permitido la creación de una web documental interactiva que constituye uno de los outputs audiovisuales del proyecto. También se han filmado muchos de los trayectos realizados en la línea R15 (itinerario Reus, Tarragona, Barcelona) con un grupo de cinco personas, que se ha organizado como grupo de viaje. En los viajes de acompañamiento realizados, tanto con informantes individuales como con el grupo de viaje, hemos podido desarrollar profusamente la conversación abierta y las entrevistas informales a la vez que, en algunas ocasiones, tomábamos testimonio audiovisual del trayecto.

\section{LA OBSERVACIÓN DIRECTA DE LA MOVILIDAD}

La relevancia del análisis de la movilidad metropolitana está relacionada, entre otros factores, con la duración que los trayectos e itinerarios de las personas tienen en el marco de una jornada de actividad cotidiana. Como hemos podido comprobar en nuestra etnografía, el traslado entre hogar y trabajo en la RMB puede ocupar poco más de media hora o prolongarse hasta más de cuatro horas, contando los viajes de ida y vuelta. En el caso de nuestra muestra, la movilidad diaria ocupa, en más de un 50\% de los casos, más de tres horas. Se trata, pues, de un fenómeno altamente significativo, pues la movilidad reduce drásticamente la capacidad de las personas para desarrollar otras actividades de tipo familiar, barrial, comunitario, formativo o de ocio. Si bien no hemos podido desarrollar suficientemente este aspecto, debemos insistir en la importancia de evaluar el impacto del commuting desde la perspectiva familiar, su organización y sus roles (Chicoine 1998; Jenks y Neves 2000; Jirón 2008).

9 El enfoque biográfico en el análisis etnográfico de la movilidad resulta especialmente provechoso, ya que las narrativas, situadas en coordenadas específicas de espacio y tiempo, nos restituyen aspectos contextuales que tienden a estar ausentes en el análisis multisituado y móvil con el que trabajamos en los estudios de commuting (Pujadas 1992, 2000). 
¿Cuál es el significado del viaje para los commuters? ¿Se trata de un inconveniente que se debe asumir, de una molestia que se debería evitar, una rutina que se ha adquirido con el tiempo? ¿O se trata de una oportunidad, de un espacio de tiempo exclusivo y privativo de cada persona que uno usa de manera libre y sin condicionantes? Las actitudes de las personas que hemos estudiado son muy variadas, desde aquellas para quienes la consecución de un trabajo estable lejos de su lugar de residencia precipita a corto o medio plazo el cambio de residencia, hasta aquellos otros que llevan más de 25 años haciendo la ruta Barcelona-Tarragona-Barcelona, considerando que se trata de una situación sostenible. Veamos algunos testimonios:

"Cuando vine [a trabajar a Tarragona] me propuse que el viaje no me debía pesar, porque me di cuenta de que si me pesaba tendría una insatisfacción vital que no quería tener. Realmente es así. A veces me siento en el autobús y pienso: iqué bien, tengo una hora de descanso! [...] Es cierto, son unas horas muy personales en que puedes pensar" [E-6]. ${ }^{10}$

"Siempre he asumido que esta era mi forma de vida. No me he impuesto nunca ningún freno a hacer esto, a hacer el esfuerzo de desplazarme a Barcelona para estudiar, para trabajar, para lo que sea... No tienes la vida familiar que tendrías si trabajases aquí [en Tarragona], a las cinco en casa. Eso lo pierdes, pero es el precio que has de pagar por el tipo de vida que has escogido" [E-29]. ${ }^{11}$

"La hora del coche por la tarde me la tomo como una hora que me sirve para relajarme y para desconectarme de los problemas del trabajo... Sin embargo, la hora de coche de la mañana me sirve para despejarme, para oír noticias y saber lo que pasa en el mundo" [E-30]. ${ }^{12}$

Se trata de tres testimonios muy representativos de las actitudes de los commuters. No están dispuestos a renunciar (o no pueden hacerlo por condicionantes familiares, o por los costos que supone el cambio de vivienda) a su estilo de vida residencial, ni tampoco desean sacrificar su carrera profesional. Por otro

10 Profesora universitaria. Vive en el ensanche de Barcelona y trabaja en la URV (Tarragona). Viaja casi a diario desde hace más de 25 años. Emplea 3 horas diarias en viajar. Usa el bus.

11 Ingeniero técnico de telecomunicaciones. Vive en Tarragona y trabaja en la Zona Franca de Barcelona en una empresa de infraestructuras y obras públicas. Realiza diariamente el viaje desde hace 17 años. Sus trayectos diarios de ida y vuelta duran algo más de cuatro horas. Viaje multimodal: coche particular, tren y bus de empresa en el viaje de ida, al revés a la vuelta.

12 Profesora universitaria. Vive en Deltebre, a $80 \mathrm{~km}$ de Tarragona. Viaja en coche particular. Hace tan solo dos años que realiza esta ruta. Su viaje de ida y vuelta tiene una duración de menos de dos horas. Viajar en tren le supondría doblar el tiempo de viaje. 
lado, comprobamos que los trayectos no son generadores de estrés. Se trata de un tiempo para relajarse, para leer, charlar, conectarse con el teléfono móvil o, simplemente, un tiempo de descanso. Muy pocos commuters consideran que los trayectos sean un tiempo perdido, vacío (Jain y Lyons 2008; Lyons, Jain y Holley 2007; Lyons y Urry 2005).

Todos los veteranos del commuting reconocen que, si su situación ha sido sostenible a lo largo del tiempo, es porque han tenido apoyo familiar o parental para compensar sus ausencias del ámbito doméstico. En los tres casos citados, la dimensión familiar operaba de tres formas diferentes. E-6 consolidó su posición en Tarragona en una época en que su madre, residente en Barcelona, requería cuidados y, más adelante, cuando su hija se hizo adolescente, se negó a abandonar su ciudad. En el caso de E-29 es la esposa quien, siendo ingeniera como él, ha optado por deslocalizar su trabajo en forma de tele-trabajo, para poder hacer frente al cuidado de los hijos. En el caso de E-30, persona de menos de 30 años, la opción de mantener su residencia en su pueblo originario se orienta a poder contar con el apoyo de su entorno familiar, especialmente de su madre, a lo largo de su etapa reproductiva, para no obstaculizar su carrera profesional que inicia ahora y que no podría desarrollar en su pueblo.

La observación de los desplazamientos nos permite comprobar las diferentes actitudes que las personas adoptan durante el tiempo del viaje. En concreto, viajar en tren no es simplemente un movimiento a lo largo de una línea predeterminada, es también un arte, el arte del viaje en tren (Watts 2008). Vemos a una mayoría de viajeros abstraídos en lecturas y juegos electrónicos, escuchando música y desconectados de su entorno inmediato, a otros trabajando, a muchos durmiendo y, todavía, algunos más desarrollando animadas conversaciones. La literatura sobre movilidad nos ofrece casos extremos, como es el caso de las personas que combinan la conducción del coche particular con la realización de gestiones telefónicas o la revisión de expedientes, esto es, usan el coche como lugar de trabajo (Laurier 2004).

Con base en las evidencias documentadas en nuestra etnografía ferroviaria, comprobamos como la mayoría de profesionales y personas de perfiles más calificados tiende a evitar dejarse inmiscuir en grupos de sociabilidad, que suele ser el espacio de tránsito que da pie a reuniones de grupos y tertulias. ${ }^{13}$ Poseen estrategias bien definidas del uso de tiempo de viaje, un tiempo

13 Existe, sin duda, una clara divisoria en las actitudes hacia el viaje de acuerdo con el grado de calificación socio-profesional. Como señalan Watts y Lyons (2007), el tiempo de viaje constituye un anatema, ya que supone una pérdida de tiempo y de dinero y constituye un tiempo inactivo; por ello, el modelo ideal de transporte tiende a minimizar los tiempos de viaje. Esta generalización, criticada por estos autores, tampoco se ha corroborado en nuestra investigación, y hemos podido comprobar el uso creativo de los tiempos de viaje. La ecuación, a mayor velocidad mayor confort en el transporte, no es validada por las actitudes de muchos viajeros, especialmente los de capas sociales medias [continua] 
que posee un valor estratégico, adaptado a cada circunstancia: completar un trabajo, leer la prensa o una novela, oír música y relajarse, dormir o, a veces, seguir la conversación de un grupo próximo y conocido. ${ }^{14}$ La privacidad que desean les lleva a elegir un vagón en el que saben que no viaja la gente conocida $y$, en nuestra muestra, hemos encontrado hasta seis ejemplos de personas que cambian de medio de locomoción para garantizar la plena y libre disponibilidad de su tiempo de viaje. ${ }^{15}$

Existen, sin embargo, diversas formas de sociabilidad entre los usuarios diarios de los transportes públicos, especialmente del ferrocarril. Hemos podido identificar tres formas básicas de sociabilidad ferroviaria. En primer lugar, grupos constituidos previamente y al margen del viaje: amigos y vecinos que coinciden en el tren, compañeros de trabajo o estudios que realizan la misma ruta o parejas que viajan juntas.

Un segundo grupo está constituido por redes de usuarios, organizados informalmente para hacer frente a las disfunciones del servicio ferroviario y que se movilizan contra la falta de soluciones frente a la impuntualidad y a las averías constantes que sufren los convoyes. Aunque de manera informal, estos usuarios constituyen una red activa y todos los implicados poseen los contactos de teléfonos móviles del resto, que son usados para convocar acciones y protestas. Estas redes se tejen, sin duda, al calor de los viajes, aunque sus miembros no practican normalmente un acompañamiento mutuo en todos los viajes, sino que se buscan en casos puntuales. ${ }^{16}$

y bajas. El tiempo de viaje, para muchos, no es un tiempo vacío, sino un espacio propio, que administran de manera creativa (Lyons y Urry 2005).

14 Entre los commuters, especialmente entre los usuarios de ferrocarril, existe la costumbre de viajar siempre en lugares prefijados. Este hecho es observable sobre todo cuando observamos a grupos de viaje. El que denominaremos el "Grupo de Isabel" (línea R15, Reus-Barcelona) viajaba siempre a la ida en la parte delantera del primer vagón, mientras que a la vuelta lo hacía en la parte trasera del convoy. Esta práctica facilita que los miembros del grupo puedan encontrarse y, aún más, porque los primeros en llegar al vagón guardan sitios para el resto del grupo. Resulta interesante esta práctica de apropiación de un espacio público.

15 Un ejemplo de esto nos lo proporciona un grupo de viaje constituido por profesorado de la Facultad de Letras de la URV, residente en Barcelona. Durante más de 30 años este grupo viajaba todos los días en tren a primera hora de la mañana, situándose en el tercer vagón del convoy. De acuerdo con una regla no escrita, cualquier miembro del grupo que desease estar solo para realizar alguna tarea (como preparar una clase, acabar de corregir ensayos de alumnos), se situaba en otro vagón del tren. Esto no impedía saludar a los colegas en la estación de inicio y reunirse con ellos en la estación de Tarragona, para ir en grupo hasta la facultad. Irónicamente, varios de los miembros del grupo nos comentaban que constituían un grupo de presión: "allí se forjaron candidaturas al decanato de la facultad y al rectorado" [E-18].

16 Algunos ejemplos de estas redes de usuarios son: Plataforma d'Usuaris de Renfe Portbou-Barcelona (ver < http://www.usuaris-renfe.com/ >), Renfadats (ver < https:/www.facebook.com/Renfadats/ >, $<$ https://twitter.com/renfadats $>,<$ https://twitter.com/hashtag/renfadats $>$ ), Plataforma Trens Dignes Terres de l'Ebre-Priorat (ver < https://trensdignesebre.wordpress.com/ >), y Renfadats Garraf (ver $<$ htt ps://plus.google.com/1 $10699929949222800835>$ >). 
Un tercer tipo de sociabilidad ferroviaria es el que se forja como resultado de la mutua voluntad de sus integrantes de practicar un acompañamiento mutuo, como resultado de su reiterado encuentro en un medio de transporte. Está constituido por personas sin antecedentes previos de relación y cuyo único vínculo es el viaje diario a lo largo de una ruta. Se trata de grupos de composición inestable, que sufren a menudo altas y bajas, cuando cambia el destino, la ocupación, los horarios laborales, o bien se decide cambiar de medio de transporte. La fragilidad en su composición se fundamenta también en los roces y malentendidos que se producen en la relación, lo que supone también la separación de alguno de sus miembros. En un sentido estricto, dada la ausencia a priori de cualquier dimensión instrumental en la relación, se trata de grupos de amistad o sociabilidad. A lo largo de la ruta entre Tarragona y Barcelona hemos observado a varios de estos grupos, si bien hemos realizado el seguimiento sistemático de tan solo uno de ellos. Como sugiere Watts, en su etnografía sobre trayectos ferroviarios en la región de Lancaster, los vagones de tren suelen presentar ambientes diferenciados, silenciosos unos, ruidosos y llenos de vida, conversación e interacción otros (Watts 2008).

Entre noviembre de 2015 y mayo de 2016 viajamos periódicamente desde Tarragona a Barcelona-Sants en el tren de la línea R15 que sale de Tarragona a las 7,00 de la mañana. Isabel, que toma el tren 17 minutos antes en Reus, guarda asientos para Mireia, Anabel y Carmen, que suben en Tarragona, y Ángel, que toma el tren en la siguiente estación, Altafulla, a las 7,12. El tren llega a su destino, salvo retrasos, a las 8,10. Estas cinco personas constituyen un "grupo de sociabilidad ferroviaria". Tienen una cuenta colectiva de WhatsApp en que se transmiten mensajes para localizarse en el convoy, cuando el vagón usado por defecto, el primero, está ocupado. Durante el viaje suelen charlar, aunque todos tienen a mano el teléfono móvil como alternativa para abstraerse del grupo. Sin embargo, a esa temprana hora de la mañana los vagones son bastante silenciosos y se respeta el descanso de los viajeros (Watts 2008: 722).

Ángel combina dos grupos de sociabilidad, el de Isabel y otro de composición exclusivamente masculina. Es bastante frecuente que en los viajes de ida (Altafulla-Barcelona) viaje con el grupo masculino y que comparta el viaje de vuelta con el grupo femenino. Existen otras dos viajeras que acompañan al grupo menos regularmente. Una de ellas es Julia, que solamente viaja dos días por semana, la otra es Pilar, que circunstancialmente viaja con el grupo de Isabel, ya que suele hacerlo con otras dos personas que buscan silencio y tranquilidad en el viaje.

Viajando reiteradamente en el mismo trayecto y a la misma hora, se puede percibir que la inmensa mayoría de los viajeros son conocidos o reconocidos por los viajeros habituales. Aunque no exista una forma de sociabilidad generalizada, más allá de cada grupo constituido, sí es cierto que se van tejiendo a 
lo largo del tiempo redes de relaciones que permiten una conectividad social a varios niveles de densidad. Por otro lado, la ruptura de algunos de los grupos constituidos por desavenencias hace que, aunque exista evitación y no se saluden, existan viajeros de los que otras personas saben toda su vida y milagros. Este entorno social denso explica, en parte, la facilidad con la que se pueden constituir grupos de activismo frente a las deficiencias del servicio ferroviario. Sin embargo, las redes personales que hemos podido etnografiar están compuestas por numerosos empleados de las dos empresas públicas que prestan el servicio ferroviario, Renfe (convoyes) y Adif (infraestructuras y estaciones), que no participan en movilizaciones activistas.

En el trayecto de ida, el grupo suele hablar poco, a pesar de que las cuatro integrantes habituales se sientan juntas. Algunas duermen y otras mantienen conversaciones en voz baja, otros consultan sus dispositivos móviles. Se trata de un doble viaje en tiempo real: el físico y el virtual (Urry 2003). Luego, a lo largo de la jornada de trabajo, la actividad de contacto virtual, vía WhatsApp, continuará, hasta el momento próximo a tomar el tren de vuelta. El viaje de vuelta, salvo imprevistos laborales de alguno de los miembros del grupo, o por alteración horaria de Renfe, se inicia a las 14,33 en un convoy de la misma línea R15. En el viaje de vuelta la primera en subir en la Estación de Francia, origen del viaje, es Mireia, quien es la encargada de guardar sitio en el convoy a su paso por Barcelona-Gràcia hasta Barcelona-Sants, donde suben Isabel, Anabel, Carmen, Ángel y algunos días Julia. Mireia suele enviar de dos a cinco mensajes al grupo de WhatsApp en ese corto trayecto, anunciando las incidencias: el modelo de tren que circula, la cantidad de gente que ocupa el tren y el lugar exacto donde está ella situada que, por defecto, es la última fila del último vagón. Según el modelo de convoy, el grupo suele elegir para sentarse los extremos de los vagones, donde hay cuatro asientos encarados con una mesita en medio. Es una manera de estar agrupados, estimulando la comunicación y el diálogo. Es también una manera de tomar posesión física del vagón, convirtiéndolo en un lugar (Watts 2008).

Más allá de la sociabilidad cotidiana, a lo largo de estos meses de acompañamiento, hemos podido asistir "on the move" a la celebración de los cumpleaños de varios de los miembros del grupo, junto a otras efemérides. En el trayecto Barcelona-Tarragona del día 16/12/2015 se realizó una comida de Navidad en que participaron las cinco personas habituales del grupo, más dos empleados de Adif, viajeros y conocidos habituales, más el etnógrafo y el cameraman que registró todo el evento. Esta comida ritual, que es similar a las denominadas "comidas navideñas de empresa", muestra el grado de institucionalización que va adquiriendo el grupo poco a poco. El 2 de febrero se celebró la comida de Carnaval también en el mismo trayecto de tren y el 1 de marzo el cumpleaños de Mireia. Cualquier disculpa es buena para dar sentido a la pertenencia al grupo y para romper la rutina. 
Junto a la dimensión corporal del viaje físico, debemos insistir en la dimensión virtual que la acompaña y refuerza. Durante los siete meses de acompañamiento del grupo sus miembros intercambiaron más de 7000 mensajes de WhatsApp. Esto confirma la hipótesis de Urry (2003), de acuerdo con la que existe una correspondencia entre la sociabilidad física, corporal, materializada en los desplazamientos diarios y, por otro lado, la conectividad mediante las redes sociales de los dispositivos móviles. Gracias a este medio de comunicación, pudimos saber que la noche de Año Nuevo Isabel tuvo que ser hospitalizada por una insuficiencia respiratoria, que el 9 de febrero Ángel tuvo que pedir la baja por una bronquitis aguda, que el día 10 de mayo nació el primer nieto de Ángel o que el pasado 16 de mayo murió la mascota de Mireia.

Sin embargo, debemos destacar que, más allá de la circulación de este tipo de mensajes que expresan la amistad y solidaridad de los miembros del grupo, la mayoría de mensajes que intercambian a diario son de tipo logístico, relacionados con la puntualidad del tren, el tipo de vagones del convoy, quién viaja y quién no cada día, quién llega tarde; también existen abundantes mensajes relacionados con las convocatorias de huelga ferroviarias y la manera como pueden afectar a sus desplazamientos. Otros motivos frecuentes de intercambio de mensajes son compartir fotografías, noticias de prensa o chistes gráficos. La actualidad política ocupa muy pocas veces las conversaciones del grupo, pero cuando lo hace suele implicar algunos roces, ya que existen puntos de vista e ideologías bastante contrastadas. Por eso, suelen evitar debatir estos temas.

\section{DE LA OBSERVACIÓN PARTICIPATIVA DE LOS VIAJES AL SOMBREADO DE LOS VIAJEROS}

La técnica del "sombreado" (shadowing, en inglés) la hemos practicado en una versión poco intrusiva con un número reducido de informantes que, por sus actividades profesionales, actitud y disponibilidad eran adecuados para su aplicación. Hemos querido evitar trascender cualquier línea roja, que supusiera romper el código ético de la etnografía y, todavía menos, que generara desasosiego entre las personas implicadas. De acuerdo con sus precursores (Wolcott 1973; Czarniawska 2014; Gaggiotti 2010, 201 1; Jirón 2007, 2008, $2011),{ }^{17}$ esta técnica de campo consiste en hacer un seguimiento sistemático a lo largo de jornadas completas de las actividades desarrolladas por una persona, incluyendo sus desplazamientos. La jornada empieza en el domicilio

17 Si bien B. Czarniawska (2014) encuentra en el relato del novelista norteamericano Truman Capote A Day's Work (Capote 1997 [1981]) el primer antecedente y la inspiración de la técnica del sombreado, no existe ninguna duda que el precedente directo de este enfoque se debe a la investigación del antropólogo H. Wolcott (1973) sobre los directores de escuela. 
del informante-colaborador, se extiende a lo largo de todo el día, tanto en el trabajo como en el resto de asuntos que la persona debe gestionar y resolver y acaba de noche, cuando el individuo se retira a su casa a descansar. Esta larga convivencia tiene como finalidad ver cómo la persona se desenvuelve en las diferentes esferas de actividad, pero también cómo cada sujeto, a la vez que desarrolla corporalmente ciertas actividades in situ, no deja de estar conectado con otras personas de manera virtual por medio de los dispositivos móviles o de Internet, en los medios familiar y laboral. Durante la jornada, la relación entre observado y observador reviste modalidades diferentes, tanto de intensa interacción como de relación autónoma y distanciada, en que el investigador simplemente observa y escribe sus notas de campo o toma fotografías.

En nuestra experiencia de sombreado, que nunca se ha producido antes de haber consolidado una relación personal con las personas analizadas, hemos realizado viajes de acompañamiento de casa al trabajo y a la inversa, les hemos acompañado a salidas y movilidades vinculadas al abastecimiento del hogar, hemos tomado café, comentando detalles surgidos de las entrevistas formales y las conversaciones informales (Gaggiotti 2011; Jirón 2011; Novoa 2015). Además, siempre que ha sido posible, hemos hablado del tema con el resto de miembros de la familia, para contrastar las opiniones sobre la movilidad y el impacto que ésta tiene sobre la organización de la vida doméstica y familiar. En algunas ocasiones, cuando las circunstancias lo han permitido, hemos buscado esa misma contrastación con compañeros o compañeras de trabajo de cada sujeto.

La secuencia de nuestra relación con cada una de las personas sombreadas ha sido, con pocas excepciones, ésta: (1) contacto por medio de algún conocido o amigo, aproximación informal al tema en casa del amigo o de la misma persona, o bien en un espacio público; (2) entrevista formal (EPOB), filmada, de unos 45 minutos de duración media; (3) transcripción de la entrevista; (4) nuevo encuentro para comentar la entrevista transcrita y para visionar un clip de 4-5 minutos con las secuencias grabadas más significativas; (5) negociación del sombreado, que se inicia siempre con algún viaje de acompañamiento; (6) el sombreado, que tiene varios escenarios, nunca se realiza a partir de un seguimiento exhaustivo de un día completo, sino en varias ocasiones, viajes de ida, de vuelta, encuentros en casa y, eventualmente, visita del trabajo y conversación informal o comida en el ámbito laboral; (7) retorno a cada persona del material recogido (por escrito y audiovisual, más el análisis realizado).

La primera experiencia de sombreado la realizamos con Isabel y Luís, una pareja de ingenieros forjada en la movilidad ferroviaria hace 17 años. Ella vivía en Reus, él en Tarragona, los dos trabajaban en Barcelona, se conocían de vista en el tren y un día coincidieron en el ascensor de un edificio de oficinas, se reconocieron de haberse visto en el tren y entablaron una relación personal que derivó en su emparejamiento. Se casaron al cabo de unos años, instalándose en 
Tarragona. Pasado un tiempo ella cambió su forma de trabajar en la empresa, pasando del viaje diario al tele-trabajo, que le permite trabajar desde casa, desplazándose a la sede de su empresa dos o tres veces al mes, cuando debe entregar algún proyecto finalizado o entrevistarse con algún cliente. Trabaja en el ámbito de la ingeniería informática, elaborando software. Tienen dos hijos y ella, desde el hogar, gestiona su trabajo y las actividades domésticas y reproductivas.

Él viaja diariamente a Barcelona, en un complejo viaje multimodal, en coche hasta Torredembarra, luego en tren hasta Barcelona y en autobús de empresa hasta el polígono de Zona Franca. Dos horas de viaje. Esta inversión de tiempo diaria condiciona en gran manera su vida personal y familiar: "[...] esto hace que el resto de tu vida gire alrededor de los horarios y el esfuerzo que te obliga a hacer el hecho de gastar cuatro horas de tus 24 sólo para desplazarte al trabajo" [E-29].

Hay muchas cosas que el commuter no puede hacer en su vida diaria: "Acostarse tarde lo debes evitar. Aunque te apetezca ver la película que están haciendo, pues, hombre un día sí lo puedes hacer, pero no lo puedes hacer por costumbre. No puedes disfrutar tanto de tu vida en casa porque llegas tarde" [E-29].

Hemos realizado un sombreado de la vida de Isabel y Luis, acompañándola a ella en su doble actividad cotidiana, laboral y doméstica, y a Luís en su larga jornada que empieza a las 6,45, cuando sale de su casa y toma el coche hasta Torredembarra, donde aparca su coche y toma el tren de la línea R15 a las 7,11, para llegar a Barcelona-Sants a las 8,10 . Si el tren cumple su horario, Luís toma el bus de empresa a las 8,20, llegando a su lugar de trabajo hacia las $8,45 .{ }^{18}$ De todos modos, tiene estudiadas todas las alternativas, si sale tarde de casa, o si el tren llega tarde. Su jornada laboral, aunque es flexible, suele extenderse hasta las 18,00. Procura tomar el tren de las 18,30. Llega de vuelta a Torredembarra hacia las 19,40 y, finalmente, a su casa a las 20,00. En total, 13 horas 15 minutos desde su salida de casa por la mañana.

Pocos espacios quedan para la vida social, para la vida familiar y doméstica. Se trata de un gran esfuerzo personal, pero que repercute en la vida de pareja y familiar. Ella relativiza la sostenibilidad de esta situación, mientras él insiste en que es una opción laboral y residencial que han adoptado, que tiene sus costos, pero que él está dispuesto a mantener. El reciente nacimiento de su segunda hija tiende a complicar todavía más este precario equilibrio entre la relación de pareja y la conciliación de la vida laboral y la personal. Este es un tema

18 Dado el carácter muy ajustado y estresante de la organización horaria del viaje de ida, nunca hemos ido a buscarle a casa para iniciar conjuntamente el viaje. Siempre nos hemos encontrado directamente en el tren, que es el mismo que toma el "Grupo de Isabel". El viaje de vuelta sí, lo hemos realizado en dos ocasiones. 
que hemos hablado conjuntamente con ellos dos y con la persona que nos los presentó, que es amiga común. Es un tema bien complejo y fuente de tensiones familiares. Tanto la precarización del mercado de trabajo como las nulas posibilidades de encontrar trabajo cerca de Tarragona a medio plazo tienden a relegar alguna toma de decisión que cambie la situación. Sin embargo, en el campo de posibilidades existe la alternativa de viajar en coche particular, que acotaría en más de 45 minutos la duración de cada desplazamiento. Estas tensiones familiares, derivadas de la articulación entre residencia y trabajo, han sido analizadas en profundidad por Green y Canny (2004) en una investigación longitudinal sobre Gran Bretaña.

El caso de Elisenda es el de una commuter atrapada por la hipoteca de su casa en Cambrils, que trabaja en Tarragona y a la que le gustaría estar viviendo en la misma ciudad donde trabaja, porque es allí donde tiene la mayoría de sus relaciones personales actuales. Viaja diariamente en su vehículo particular, desde la urbanización de Cambrils donde tiene su casa hasta el Rectorado de la Universidad, donde trabaja, situado junto a la muralla Nordeste del casco antiguo de Tarragona. La autovía A7 le permite llegar en 20 minutos. No tiene problemas de aparcamiento. Su jornada es de 9,00 a 14,00 y de 16,00 a 19,00. Al mediodía su rutina consiste en ir a un centro deportivo situado en una zona residencial y ajardinada, situada al Norte del municipio. Allí suele jugar al paddle y aprovecha para comer. Tiene amistades en Tarragona que la acompañan. Algunos días no vuelve a Cambrils y se queda a dormir en casa de una amiga. Además de su vida laboral y social, en Tarragona también realiza la mayoría de sus compras.

"Yo compré la casa en 2001. En aquel momento pensaba diferente de ahora. Pensaba que pagaba lo mismo por comprar que por alquilar y alquilando tiraba el dinero. Por eso me compré la vivienda allí, porque tenía mi familia, mi pareja y cuestiones personales que me condicionaron. Ahora estaría viviendo en Tarragona y de alquiler [...] Yo tengo una hipoteca bastante alta y te das cuenta que realmente te condiciona. Además, yo no tengo un piso, tengo una casa!" [E-7]

Hemos monitorizado la vida de Elisenda por medio de varias salidas al mediodía para acompañarla desde su oficina, donde hemos sombreado el final de su jornada laboral matutina, hasta el club deportivo donde se relaja, hace ejercicio, come y se relaciona. También hemos estado en su casa, participando de sus momentos de ocio. Los sucesivos encuentros, tras haber analizado conjuntamente su $\mathrm{EPOB}$, nos han permitido ir completando información, pasando de opiniones al registro de prácticas, a narraciones de sus actividades concretas y contextualizadas. Hemos hablado del futuro y de su deseo de vender la casa, una vez la tenga pagada, para vivir de alquiler en el barrio de la Arrabasada en 
Tarragona, donde habitan sus amistades actuales y que le permitiría ir a pie al trabajo. Su vínculo con Cambrils se limita a la visita periódica a sus padres, pero sus relaciones sociales se han desplazado de lugar con el tiempo. Se centran en Tarragona y en Barcelona, donde trabajó unos años, antes de recalar en la URV.

El caso de Francisco es el de un ingeniero de caminos barcelonés que hace cinco años que trabaja en la sede leridana de una empresa eléctrica. Vivió en Lleida tres años, hasta que conoció a su actual pareja, residente en Tarragona. Han constituido su hogar en Tarragona y desde hace dos años él viaja diariamente de Tarragona a Lleida para ir a trabajar: "Yo salgo a las 6,15 de casa, clavado, estoy cerrando la puerta. Y estoy de nuevo sentado en el pupitre, en la oficina, en Lleida, a las 7,25" [E-22].

Se trata de una combinación muy eficiente de viaje mixto que incluye el uso del coche particular de Tarragona a la estación del tren de alta velocidad de Camp de Tarragona, que toma hasta Lleida (trayecto de 25 minutos) y allí con un segundo vehículo particular llega a su lugar de trabajo en un polígono industrial a las afueras de Lleida. Sale a las 6,15 de casa, toma el tren Avant a las 6,38, llega a Lleida a las 7,02 y, finalmente, a su lugar de trabajo a las 7,25 . Hemos estado con él en su lugar de trabajo y hemos conocido a algunos de sus compañeros. Con permiso de la empresa hemos tomado imágenes de él trabajando, con lo que hemos tenido oportunidad de profundizar en las rutinas y dinámicas que tiene la actividad de un ingeniero de caminos que controla el funcionamiento y las obras de mantenimiento de toda una cuenca hidrográfica. Tenemos pendiente acompañarle en alguna de las salidas in situ que realiza periódicamente a alguna de las instalaciones de la compañía en el Prepirineo y Pirineo de Lleida y de la parte Oriental de Huesca.

\section{ALGUNAS CONCLUSIONES}

La etnografía móvil desplaza el centro de interés desde el lugar (no importa tanto qué personas) hacia las personas (no importa tanto en qué lugares). Los espacios difícilmente llegan a constituirse en lugares, ya que lo que registran son esencialmente tránsitos. Los no lugares, como diría Augé (2000 [1992]), proliferan. Aquello que dota a un espacio de sentido son los usos de las personas. Y es a través de las personas como identificamos lugares, usos, apropiaciones, conflictos, refuncionalizaciones.

A partir de nuestra experiencia del estudio de la movilidad en la RMB, hemos avanzado en la comprensión de las principales variables que acompañan y delimitan los diferentes modos y estrategias de la movilidad de las personas: (1) las personas jóvenes se mueven mucho más que las mayores; (2) los hombres se mueven más que las mujeres, lo que nos remite a los sesgos que imponen los roles de género (Chicoine 1998); (3) la composición de las familias y 
las diferentes etapas del ciclo reproductivo condicionan fuertemente las estrategias de movilidad familiar; (4) la conciliación entre actividad laboral y tareas reproductivas suele ir acompañada de estrategias de búsqueda de proximidad residencial respecto a ciertos miembros de la parentela (Green y Canny 2004); (5) la ubicación residencial es la principal variable respecto a la movilidad requerida para trabajar, pero también para aprovisionarse y para el ocio; (6) la movilidad residencial se produce, en general, siguiendo un ideal de estilo de vida más que su funcionalidad en términos de minimizar distancias y costos de transporte (Green y Canny 2004); (7) aunque el uso del vehículo privado se justifica en términos de racionalidad del uso del tiempo, en la mayoría de los casos constituye una elección subjetiva tendente a evitar las rigideces de horarios del transporte público (Dant 2004; Urry 2004).

Más allá de los avances en la comprensión de la movilidad misma, nuestra investigación nos ha permitido profundizar en los requerimientos y disposiciones exigidas para la mejor observación de sujetos móviles (Jirón 2008; Novoa 2015; Watts 2008; Spinney 2011). Nuestra unidad de análisis ha requerido paradójicamente de una delimitación espacial, la RMB, si bien se trata de una unidad espacial de fronteras abiertas y porosas. Nuestro punto de partida ha sido, esencialmente, el Camp de Tarragona y, más en concreto, la ciudad de Tarragona, donde habita una mayoría de las personas cuyas movilidades hemos ido observando de manera sistemática. Sin embargo, oficialmente, Tarragona no forma parte de la RMB. Las prácticas de movilidad de nuestros sujetos, por el contrario, nos remiten una y otra vez a la RMB y a su capital. Son ellos, pues, quienes, al margen de delimitaciones oficiales, han ido fijando con sus prácticas nuevas fronteras y nuevas funcionalidades a un espacio acotado por sus tránsitos.

Si la comprensión del fenómeno macro por excelencia, la globalización, llevó a algunos de los autores más innovadores de las ciencias sociales a la recomendación de "seguir los objetos" (Wallerstein 1974; Mintz 1985; Harvey 1998 [1989]), la práctica de una etnografía móvil parte del requerimiento de "seguir a las personas". Consideramos que el "acompañamiento" de los commuters constituye la técnica etnográfica por excelencia para la observación de sus prácticas particulares en un universo móvil lleno de posibilidades. A pesar de la espectacularidad y del carácter promisorio de su planteamiento en los autores ya citados que la amparan (Wolcott 1973; Czarniawska 2007, 2014; Jirón 2008, 201 1; Gaggiotti 2010, 2011), consideramos que la técnica del sombreado presenta demasiadas líneas rojas y conflictos éticos, como para abundar en ella. Es cierto que nuestra práctica del acompañamiento puede llegar a tener muchos rasgos compartidos con aquella. Sin embargo, se trata de énfasis diferenciados, tanto a nivel ético como epistemológico. Es la persona la que marca las dinámicas e impone límites y restricciones a nuestra labor de observadores. Nuestra actitud discreta, expectante, predispuesta al 
acompañamiento ha encontrado pocas resistencias. Hemos trascendido límites en cuanto a la intimidad de nuestro acercamiento a las prácticas cotidianas de las personas, impensables en el inicio de la investigación. El tiempo, la corrección, la empatía y hasta la amistad personal, por qué no decirlo, nos han abierto puertas, a priori cerradas.

La cuestión central de nuestro planteamiento, y aquí aparece un sesgo epistemológico, no ha sido observar-prácticas-de-movilidad-de-personas, sino estudiar-prácticas-de-movilidad-con-las-personas. No hemos dispuesto de informantes, al estilo clásico, sino que hemos planteado un tema de estudio y hemos pretendido estudiarlo con ellos y ellas. No todos los commuters, ni de la misma manera, se han implicado en el proyecto de investigación, pero sí es cierto que nueve o diez personas se han erigido en partícipes de esta experiencia compartida hasta unos límites insospechados. Se ha tratado, pues, de una experiencia de trabajo colaborativo.

\section{BIBLIOGRAFÍA}

ABU-LUGHOD, Janet, 1961, "Migrant adjustment to city life: the Egyptian case", American Journal of Sociology, 67 (1): 22-32.

ALBERICH GONZÁLEZ, Joan, 2010, "La metropolitanització del territori català: una anàlisi a partir dels espais de vida de la población", Treballs de la Societat Catalana de Geografia, 69: 39-65.

ALBERICH GONZÁLEZ, Joan, y Santiago ROQUER SOLER, 2014, "El espacio de vida de la población catalana metropolitana: un análisis a partir de la 'Enquesta de Condicions de Vida i Hàbits de la Población 201 l' ", en A. López Gay et al. (comps.), XIV Congreso Nacional de Población. Sevilla, Grupo de Población de la Asociación de Geógrafos Españoles, 486-497.

APPADURAI, Arjun, 1990, "Disjuncture and difference in the global cultural economy", Public Culture, 2: 1-24.

APPADURAI, Arjun, 1991 [1986], La Vida Social de las Cosas: Perspectiva Social de las Mercancías. México, DF, Grijalbo.

AUGÉ, Marc, 2000 [1992], Los No Lugares: Espacios del Anonimato. Barcelona, Editorial Gedisa. BARNES, John A., 1954, "Class and committees in a Norwegian island parish", Human Relations, 7 (1): 39-58.

BASCOM, William R., 1955, "Urbanization among the Yoruba", American Journal of Sociology, LX (5): 446-454.

BAUMAN, Zygmunt, 2003 [2000], Modernidad Líquida. Buenos Aires, FCE.

BECK, Ulrich, 1998 [1986], La Sociedad del Riesgo: Hacia Una Nueva Modernidad. Barcelona, Paidós. 
BECK, Ulrich, 2004, "Las sociedades cosmopolitas y sus enemigos", en Ulrick Beck, La Mirada Cosmopolita o la Guerra Es la Paz. Barcelona, Paidós, 103-138.

BENJAMIN, Walter, 2005, Libro de los Pasajes. Madrid, Akal.

BOISSEVAIN, Jeremy, y J. Clyde MITCHELl (comps.), 1973, Network Analysis. La Haya, Mouton.

BOTT, Elizabeth, 1957. Family and Social Network. Londres, Tavistock.

BRUECKNER, Jan K., 2000, “Urban sprawl: diagnosis and remedies”, International Regional Science Review, 23 (2): 160-171.

BUTTERWORTH, Douglas S., 1962, "A study of the urbanization process among Mixtec migrants from Tilaltongo in Mexico City", América Indígena, 22 (3): 257-274.

CAPOTE, Truman, 1997 [1981], "Un día de trabajo", en Truman Capote (comp.), Música para Camaleones. Barcelona, Anagrama, 82-92.

CHICOINE, Nathalie, 1998, “Nouvelles dynamiques métropolitaines et stratégies spatiales de la main d'œuvre: le cas d'employées de bureau montréalaises”, en Horacio Capel y Paul-André Linteau (comps.), Barcelona-Montréal: Desarrollo Urbano Comparado/Développement urbain comparé. Barcelona, Publicacions de la Universitat de Barcelona, 51-62.

COURGEAU, Daniel, 1988, Méthodes de mesure de la mobilité spatiale: Migrations internes, mobilité temporaire, navettes. París, INED.

CZARNIAWSKA, Barbara, 2007, Shadowing and Other Techniques for Doing Fieldwork in Modern Societies. Malmö, Liber.

CZARNIAWSKA, Barbara, 2014, "Observation on the move: shadowing", en B. Czarniawska, Social Science Research: From Field to Desk. Los Angeles, Sage, 43-56.

DANT, Tim, 2004, “The driver-car”, Theory, Culture \& Society, 21 (4/5): 61-79.

DEBORD, Guy, 1999 [1958], “Teoría de la deriva”, en Guy Debord, Internacional Situacionista, vol. 1, La Realización del Arte. Madrid, Literatura Gris, 50-53.

DURÁN SEGURA, Luis Armando, 2011 , "Miradas urbanas sobre el espacio público: el flâneur, la deriva y la etnografía de lo urbano", Reflexiones, 90 (2): 137-144.

EPSTEIN, A. L., 1958, Politics in an Urban African Community. Manchester, Manchester University Press.

EPSTEIN, A.L., 1967, "Urbanization and social change in Africa", Current Anthropology, 8 (4): 275-295.

FEATHERSTONE, Mike (comp.), 1990, Global Culture: Nationalism, Globalism, and Modernity. Londres, Sage.

FOX, Richard G., 1977, Urban Anthropology: Cities in Their Cultural Settings. Englewood Cliffs, NJ, Prentice-Hall.

FRANQUESA, Jaume, 2011 , “'We've lost our bearings': place, tourism, and the limits of the "mobility turn'”, Antipode, 43 (4): 1012-1033.

GAGGIOTTI, Hugo, 2010, "Official chronicles of corporate globalization and unofficial stories of international mobility: resisting patronage of meanings?”, Journal of Organizational Change Management, 23 (2): 157-165.

GAGGIOTTI, Hugo, 2011, "Narrating expatriation and making sense of the globalization experience", en E. Bonet et al. (comps.), Rhetoric and Narratives in Management Research. Sant Cugat, ESADE, 293-306.

GARCÍA-CANCLINI, Néstor, 1989, Culturas Híbridas: Estrategias para Entrar y Salir de la Modernidad. México, DF, Grijalbo. 
GLICK SCHILLER, Nina, Linda BASCH, and Cristina SZANTON BLANC, 1992, “Transnationalism: a new analytic framework for understanding migration", Annals of the New York Academy of Sciences, 645 (1): 1-24.

GLICK SCHILLER, Nina, y Noel SALAZAR, 2013, "Regimes of mobility across the globe", Journal of Ethnic and Migration Studies, 39 (2): 183-200.

GLUCKMAN, Max, 1958, Analysis of a Social Situation in Modern Zululand. Manchester, Manchester University Press.

GREEN, Anne E., y Angela CANNY, 2004, Geographical Mobility: Family Impacts. Bristol, Policy Press.

HANNERZ, Ulf, 1969, Soulside: Inquiries into Ghetto Culture and Community. Nueva York, Columbia University Press.

HANNERZ, Ulf, 1986 [1980], Exploración de la Ciudad. México, DF, FCE.

HANNERZ, Ulf, 1992, Cultural Complexity: Studies in the Social Organization of Meaning. Nueva York, Columbia University Press.

HANNerZ, Ulf, 1996, Transnational Connections: Culture, People, Places. Nueva York, Taylor \& Francis.

HARVEY, David, 1998 [1989], La Condición de la Posmodernidad: Investigación sobre los Orígenes del Cambio Cultural. Buenos Aires, Amorrortu Editores.

IMILÁN, Walter, Paola JIRÓN, y Luis ITURRA, 2015, "Más allá del barrio: habitar Santiago en la movilidad cotidiana”, Revista Antropologías del Sur, 3: 87-103.

JAIN, Juliet, y Glenn LYONS, 2008, “The gift of travel time”, Journal of Transport Geography, 16 (2): 81-89.

JENKS, Chris, y Tiago NEVES, 2000, "A walk on the wild side: urban ethnography meets the flâneur", Journal for Cultural Research, 4 (1): 1-17.

JIRÓN, Paola, 2007, "Unravelling invisible inequalities in the city through urban daily mobility: the case of Santiago de Chile", Swiss Journal of Sociology, 33 (1): 45$-68$.

JIRÓN, Paola, 2008, Mobility on the Move: Examining Urban Daily Mobility Practices in Santiago de Chile. Londres, LSE.

JIRÓN, Paola, 2011, “On becoming 'la sombre/the shadow'”, en M. Büscher, J. Urry y K. Witchger (comps.), Mobile Methods. Londres, Routledge, 36-53.

LASH, Scott, y John URRY, 1998, Economías de Signos y Espacio: Sobre el Capitalismo de la Posorganización. Buenos Aires, Amorrortu Editores.

LAURIER, Eric, 2004, "Doing office work on the motorway", Theory, Culture \& Society, 21 (4-5): 261-277.

LEVITT, Peggy, y Nina GLICK SCHILLER, 2004, "Perspectivas internacionales sobre migración: conceptualizar la simultaneidad”, Migración y Desarrollo, 3: 60-91.

LEWIS, Oscar, 1961, The Children of Sanchez. Nueva York, Random House.

LEWIS, Oscar, 1966, La Vida: A Puerto Rican Family in the Culture of Poverty. San Juan and New York. Nueva York, Vintage Books.

LITTLE, Kenneth, 1974, Urbanization as a Social Process: An Essay on Movement and Change in Contemporary Africa. Londres, Routledge.

LOMNITZ, Larissa Adler, 1977, Networks and Marginality: Life in a Mexican Shantytown. Nueva York, Academic Press.

LYONS, Glenn, Juliet JAIN, y David HOLLEY, 2007, “The use of travel time by rail passengers in Great Britain”, Transportation Research (Part A), 41 (1): 107-120. 
LYONS, Glenn, y John URRY, 2005, "Travel time use in the information age", Transportation Research (Part A), 39 (2): 257-276.

MARCUS, George E., 1995, "Ethnography in/of the World System: the emergence of multisited ethnography", Annual Review of Anthropology, 24: 95-1 17.

MARKS, Carole, 1991, “The urban underclass”, Annual Review of Sociology, 17: 445-466.

MAYER, Philip, 1961, Townsmen or Tribesmen: Conservatism and the Process of Urbanization in a South African City. Londres, Oxford University Press.

MERRIMAN, Peter, 2009, "Automobility and the geographies of the car", Geography Compass, 3 (2): 586-599.

MIESZKOWSKI, Peter, y Edwin S. MILLS, 1993, “The causes of metropolitan suburbanization", The Journal of Economic Perspectives, 7 (3): 135-147.

MINTZ, Sidney W., 1985, Sweetness and Power: The Place of Sugar in Modem History. Nueva York, Viking.

MITCHELL, J. Clyde, 1956, The Kalela Dance. Manchester, Manchester University Press.

MITCHELL, J. Clyde, 1969, "The concept and use of social networks", en J.C. Mitchell (comp.), Social Networks in Urban Situations. Manchester, Manchester University Press, $1-50$.

MOLINA GONZÁLEZ, José Luis, 2005, "El estudio de las redes personales: contribuciones, métodos y perspectivas”, Empiria: Revista de Metodología de Ciencias Sociales, 10 : $71-105$.

NOVOA, Andre, 2015, "Mobile ethnography: emergence, techniques and its importance to geography”, Human Geographies, 9 (1): 97-107.

PARK, Robert E., 1952, Human Communities: the City and Human Ecology. Glencoe, IL, The Free Press.

PELLICER, Isabel, Jesús ROJAS, y Pep VIVAS-ELIAS, 2012, "La deriva: una técnica de investigación psicosocial acorde con la ciudad contemporánea”, Boletín de Antropología, 27 (44): 144-163.

PELLICER, Isabel, Pep VIVAS-ELIAS, y Jesús ROJAS, 2013, “La observación participante y la deriva: dos técnicas móviles para el análisis de la ciudad contemporánea. El caso de Barcelona", EURE, 39 (116): 119-139.

PINK, Sarah, 2008, "Walking with video”, Visual Studies, 22 (3): 240-252.

PRYSTHON, Ângela Freire, 2007, "A grande aventura urbana”, Galáxia: Revista do Programa de Pós-Graduação em Comunicação e Semiótica, 13: 151-153.

PUJADAS, Joan Josep, 1992, El Método Biográfico: Las Historias de Vida en Ciencias Sociales. Madrid, Centro de Investigaciones Sociológicas.

PUJADAS, Joan Josep, 2000, "El método biográfico y los géneros de la memoria”, Revista de Antropología Social, 9: 127-158.

PUJADAS, Joan Josep, 2011 , "Los claroscuros de la etnicidad: el culturalismo evaluado desde la óptica de la cohesión social y la ciudadanía”, en P. Palenzuela y A. Olivi (comps.), Etnicidad y Desarrollo en los Andes. Sevilla, Universidad de Sevilla, 25-51.

ROCHA, Ana Luiza Carvalho da, y Cornelia ECKERT, 2003, "Etnografia de rua: estudo de antropologia urbana", Iluminuras, 4 (7): 1-22.

SAFA BARrAZA, Patricia, 1998, Vecinos y Vecindarios en la Ciudad de México: Un Estudio sobre la Construcción de Identidades Vecinales en Coyoacán. México, DF, Ángel Porrúa.

SALAZAR, Noel B., 2010, “Towards an anthropology of cultural mobilities”, Crossings: Journal of Migration and Culture, 1 (1): 53-68. 
SANTOS, Hugo Miguel, Manuela Martinho FERREIRA, y Sofia Marques da SILVA, 2014, "Uma (multi)etnografia urbana das culturas juvenis gays e as suas práticas de conformação, respeitabilidade e subversão”, apresentado en XIV Colóquio Ibérico de Geografia, junio de 2014.

SHELlER, Mimi, y John URRY, 2006, “The new mobilities paradigm”, Environment and Planning (A), 38 (2): 207-226.

SPINNEY, Justin, 2011, "A chance to catch a breath: using mobile video ethnography in cycling research", Mobilities, 6 (2): 161-182.

URRY, John, 2003, "Social networks, travel and talk", The British Journal of Sociology, 54 (2): 155-175.

URRY, John, 2004, “The 'system' of automobility”, Theory, Culture \& Society, 21 (4-5): 25-39.

URRY, John, 2008, "Governance, flows, and the end of the car system?", Global Environmental Change, 18 (3): 343-349.

VAlentine, Charles A., 1968, Culture and Poverty: Critique and Counter-Proposals. Chicago, The University of Chicago Press.

WALlERSTEIN, Immanuel, 1974, The Modern World-System. Nueva York, Academic Press.

WARE, Caroline F., 1935, Greenwich Village, 1920-1930: A Comment on American Civilization in the Post-War Years. Nueva York, Harper and Row.

WATTS, Laura, 2008, “The art and craft of train travel”, Social \& Cultural Geography, 9 (6): 711-726.

WATTS, Laura, y Glenn LYONS, 2007, “Travel remedy kit: interventions into train lines and passenger times", presentado en seminario del Centre for Transport \& Society, 18 de noviembre de 2007, disponible en < http://eprints.lancs.ac.uk/10658/ > (última consulta en junio de 2018).

WHYTE, William Foote, 1943, Street Corner Society. Chicago, The University of Chicago Press.

WILlis, Paul, 1988 [1978], Aprendiendo a Trabajar: Cómo los Chicos de la Clase Obrera Consiguen Trabajos de Clase Obrera. Madrid, Akal.

WIRTH, Louis, 1938, "Urbanism as a way of life", American Journal of Sociology, 44 (1): 1-24. WOLCOTT, Harry F., 1973, The Man in the Principal's Office: An Ethnography. Walnut Creek, CA, Altamira Press.

Receção da versão original / Original version

Receção da versão revista / Revised version

Aceitação / Accepted
$2016 / 12 / 11$

$2017 / 07 / 04$

$2018 / 05 / 15$ 